Z Epileptol 2011 $\cdot 24: 223-223$

DOI 10.1007/s10309-011-0186-6

C) Springer-Verlag 2011

I. Helbig, S. von Spiczak, J. Albers, H. Muhle, U. Stephani

\title{
Verführt die neue Klassifikation zu genetischem Wunschdenken?
}

den, dass z. B. bei der häufigen CAE im Großteil der Patienten starke genetische Risikofaktoren (sog. rare variants) bzw. monogene Risikofaktoren vermutet werden, die für den einzelnen Patienten von Bedeutung sind. Dies kann eventuell bei der Patientenberatung das Missverständnis mit sich bringen, dass ein fälschlich hohes Risiko für Familienmitglieder angenommen wird und dass die CAE als „Erbkrankheit“ oder „Ein-Gen-Erkrankungen“ (z. B. mit Erbgängen nach Mendel-Regeln) aufgefasst wird.

Es gibt mehrere Modelle der genetischen Architektur, die mit der hohen Heritabilität der CAE kompatibel sind. In vielen häufigen Erkrankungen können oft Single Nucleotide Polymorphisms (SNPs) als Risikofaktoren nachgewiesen werden, die auf Populationsebene einen Teil des Risikos erklären. Auf individueller Patientenebene stellen diese genetischen Faktoren lediglich milde Risikofaktoren dar, die das Risiko für die Erkrankungen um das maximal Zwei- bis Dreifache erhöhen. Andere Mechanismen wie z. B. Gen-Gen-Interaktion sind bislang unzureichend erforscht, werden aber als relevant angenommen; nur ein Teil der Heritabilität häufiger Erkrankungen lässt sich bislang durch bekannte häufige oder seltene Risikofaktoren erklären (Konzept der missing heritability [5]).

Wir möchten daher die Diskussion darüber anregen, in der Gruppe der „genetischen Epilepsien“ zwischen monogenen und komplex-genetischen Epilepsien zu trennen.
Literatur

1. Berg, A.T., et al., Revised terminology and concepts for organization of seizures and epilepsies: report of the ILAE Commission on Classification and Terminology, 2005-2009. Epilepsia. 2010 Apr;51(4):676-85

2. Berkovic, S.F., et al., Epilepsies in twins: genetics of the major epilepsy syndromes. Ann Neurol, 1998. 43(4): p. 435-45.

3. Lennox, W.G., The heredity of epilepsy as told by relatives and twins. J Am Med Assoc, 1951. 146(6): p. 529-36.

4. Helbig, l., et al., Navigating the channels and beyond: unravelling the genetics of the epilepsies. Lancet Neurol, 2008. 7(3): p. 231-45.

5. Manolio, T.A., et al., Finding the missing heritability of complex diseases. Nature, 2009. 461(7265): p. 747-53.

\section{Korrespondenzadresse}

\section{Helbig}

Klinik für Neuropädiatrie,

Christian-Albrechts-Universität zu Kiel

Schwanenweg 20, 24105 Kiel

i.helbig@pedneuro.uni-kiel.de 